\title{
Characterization of microRNA expression in serous ovarian carcinoma
}

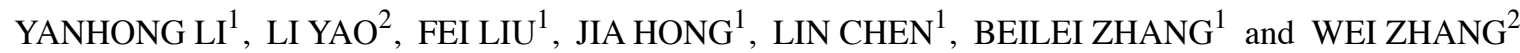 \\ ${ }^{1}$ Department of Gynecology and Obstetrics; ${ }^{2}$ The Helmholtz Sino-German Research Laboratory for Cancer, \\ Department of Pathology, Tangdu Hospital, The Fourth Military Medical University, Xi'an, Shaanxi 710038, P.R. China
}

Received February 17, 2014; Accepted May 21, 2014

DOI: $10.3892 /$ ijmm.2014.1813

\begin{abstract}
Serous ovarian cancer is a major gynecologic malignancy with a poor 5-year survival rate. However, little is known regarding the behavior and genetics of ovarian tumorigenesis. MicroRNAs (miRNAs) have been shown to be dysregulated in ovarian carcinomas. To assess the miRNA expression profiles in serous ovarian cancer, we defined the patterns of miRNA expression in 100 formalin-fixed, paraffin-embedded ovarian cancer tissues blocks as well as 50 corresponding normal oviduct tissues using miRNA microarray. MiRNA expression profiling showed that 63 miRNAs were downregulated and 43 miRNAs were upregulated in serous ovarian cancer tissues compared with control tissues. The expression of five dysregulated miRNAs was validated using quantitative polymerase chain reaction (RT-qPCR). GO term and pathway analysis revealed that the biological process of the cell cycle was significantly enriched and the MAPK signaling pathway was highly involved in the progression of ovarian cancer. The results suggested that the aberrant expression of miRNAs is involved in ovarian carcinogenesis and thus these miRNAs may function as diagnostic and prognostic biomarkers.
\end{abstract}

\section{Introduction}

Ovarian cancer is the most lethal of the gynecologic malignancies worldwide (1). Epithelial ovarian cancer, which accounts for $90 \%$ of ovarian cancer, is a heterogeneous group

Correspondence to: Professor Yanhong Li, Department of Gynecology and Obstetrics, Tangdu Hospital, The Fourth Military Medical University, No. 1 Xin Si Road, Xi'an, Shaanxi 710038, P.R. China

E-mail: lyhzhw@fmmu.edu.cn

Professor Wei Zhang, The Helmholtz Sino-German Research Laboratory for Cancer, Department of Pathology, Tangdu Hospital, The Fourth Military Medical University, No. 1 Xin Si Road, Xi'an, Shaanxi 710038, P.R. China

E-mail: w_zhang66@yahoo.com

Key words: microRNA, ovarian cancer, microarray, biomarker of carcinoma and encompasses several histologic subgroups, each with their own organic molecular genetic events, such as Brenner, endometrioid, mucinous or serous carcinoma $(2,3)$. Among them, the serous type is responsible for $75-80 \%$ of epithelial ovarian malignancies, and the majority of serous carcinomas are at an advanced stage at diagnosis (4). However, high-grade serous ovarian cancer usually exhibits very high tumor heterogeneity, altered gene expression and genome instability (5), which increases the complexity of the ovarian cancer pathogenesis and complicates the search for signatures that characterize this disease. Therefore, a better understanding of molecular alterations in serous ovarian carcinoma is necessary to identify novel targets for early detection and improved treatment.

MicroRNAs (miRNAs) are a new class of endogenous non-coding single-stranded small RNAs, 22 nucleotides in length, that regulate mRNA function by perfect or partial base pairing with the complementary mRNA $(6,7)$. The binding of miRNAs to their target mRNAs leads to a decrease in the stability of mRNA or translational inhibition. Most miRNAs possess oncogenic or tumor suppressor activity and can modulate diverse biological processes, including development, drug chemoresistance, metabolism, cell proliferation and apoptosis (8-10). As miRNA expression is tissue-specific, detectable in blood (11), and correlates with clinical cancer behavior (12), miRNAs may be useful as potential valuable diagnostic and prognostic markers.

It has been reported that ovarian cancer is closely associated with multistep changes in the genome, particularly the expression and function of various miRNAs $(13,14)$. Using global profiling $(15,16)$ and candidate gene $(11,14,17)$ approaches, up- and downregualted miRNAs have been identified in ovarian carcinoma samples. The aberrant expression of miRNAs has also been associated with tumor histology (18), response to survival (19) and therapy $(20,21)$. Although previous studies have shown great potential for the use of miRNA in diagnosis, prognosis, and therapy in ovarian cancer $(22,23)$, the precise association between aberrant miRNA expression and the clinicopathology of ovarian cancer has not been thoroughly evaluated.

In this study, we investigated miRNA expression in matched pairs of primary serous ovarian tumors and normal oviduct tissues using microarray and attempted to identify miRNAs capable of predicting the clinical diagnosis and prognosis. 
Table I. Clinicopathological characteristics of cases of ovarian carcinoma $(n=100)$.

\begin{tabular}{|c|c|c|c|c|c|c|c|c|c|c|c|}
\hline \multirow[t]{2}{*}{ Age } & \multirow[t]{2}{*}{ No. } & \multicolumn{4}{|c|}{ Clinical stage } & \multicolumn{2}{|c|}{ Diff grade } & \multicolumn{2}{|c|}{ LNM } & \multicolumn{2}{|c|}{ CA125 (U/ml) } \\
\hline & & I & II & III & IV & WD & $\mathrm{MD} / \mathrm{PD}$ & Yes & No & $\geq 200$ & $<200$ \\
\hline $21-30$ & 3 & 1 & 0 & 2 & 0 & 1 & 2 & 1 & 2 & 1 & \\
\hline $31-40$ & 7 & 1 & 1 & 5 & 0 & 0 & 7 & 4 & 3 & 2 & 2 \\
\hline $41-50$ & 20 & 3 & 2 & 11 & 4 & 1 & 19 & 9 & 11 & 18 & 5 \\
\hline $51-60$ & 45 & 4 & 13 & 21 & 7 & 4 & 41 & 15 & 30 & 30 & 2 \\
\hline $61-70$ & 21 & 2 & 1 & 12 & 6 & 1 & 20 & 10 & 11 & 15 & 15 \\
\hline $71-80$ & 3 & 0 & 0 & 2 & 1 & 0 & 3 & 2 & 1 & 3 & 6 \\
\hline $81-90$ & 1 & 0 & 0 & 1 & 0 & 1 & 0 & 1 & 0 & 1 & 0 \\
\hline Total & 100 & 11 & 17 & 54 & 18 & 8 & 92 & 42 & 58 & 70 & 30 \\
\hline
\end{tabular}

WD, well differentiated; MD, moderately differentiated; PD, poorly differentiated; Diff grade, differentiation grade; LNM, lymph node metastasis .

\section{Materials and methods}

Patients and clinical tissue samples. The protocol of the present study was approved by the ethics committee of the Fourth Military Medical University. A total of 150 archived formalin-fixed, paraffin-embedded (FFPE) tissue blocks of normal oviduct tissues (50 cases) and serous ovarian carcinomas (100 cases) were obtained from patients in the Department of Gynecology and Obstetrics, Tangdu Hospital, the Fourth Military Medical University (Xi'an, China). The histomorphology of all the primary tumors specimens and regional lymph nodes was confirmed with hematoxylin and eosin (H\&E) staining. Histopathological grading was evaluated in accordance with the criteria of the International Federation of Gynecology and Obstetrics (FIGO) grading system and the histological subtype of each ovarian tumor was diagnosed according to the World Health Organization classification. Clinical parameters such as gender, age, differentiation status, lymph node metastasis, serum CA125 value and TNM stage were collected (Table I).

$H \& E$ staining and immunohistochemical (IHC) assay. Standardized H\&E staining was used to evaluate the morphology of ovarian tumor and normal tissues. To confirm the characteristics of ovarian cancer, IHC was performed using the avidin-biotin-peroxidase method on the ovarian cancer tissues and normal oviduct tissues. All the specimens were fixed with $4 \%$ paraformaldehyde and embedded with paraffin. Sections $(4 \mu \mathrm{m})$ were cut and transferred to glass slides coated with $100 \mathrm{~g} / \mathrm{l}$ polylysine. The primary antibodies were diluted in PBS: Anti-cytokeratin 7 (CK7) (1:100; Abcam, Cambridge, UK). An immunohistochemical analysis was performed on the paraffin-embedded sections. The sections were autoclaved for $10 \mathrm{~min}$ at $121^{\circ} \mathrm{C}$ for antigen retrieval. An EnVision kit (Dako, Glostrup, Denmark) was used, and the IHC staining was performed according to the manufacturer's instructions. The negative control was performed by replacing the primary antibody with pre-immune murine serum.

RNA extraction. Total RNA was extracted from paraffin-embedded tissues and homogenized in TRIzol reagent (Invitrogen Life Technologies, Carlsbad, CA, USA), in accordance with the manufacturer's instructions. RNA purity and concentration were confirmed by spectrophotometry using the NanoDrop ND-1000 (NanoDrop Technologies, Wilmington, DE, USA).

Microarray analysis. For the miRNA microarray study, eight cases of serous tumors and eight normal oviduct tissues were randomly selected and assessed. The clinical parameters of the eight serous tumors are shown in Table II. Total RNA $(50 \mu \mathrm{g})$ was processed to enrich the miRNA using a mirVana RNA Isolation kit (Applied Biosystems, Inc., Foster City, CA, USA). DNA oligonucleotide probes from the mirVana miRNA Probe Set (Applied Biosystems, Inc.), containing 739 human precursor and mature miRNA, were printed on coated glass slides in duplicate (Digital Genomics, Suwon, Korea). Additionally, $50 \mu \mathrm{mol} / 1$ probes were resuspended with 3X SSC and spotted on AttayIt SuperEpoxy2 (TeleChem Corp., Atlanta, GA, USA) under 55\% humidity using the ArrayIt SpotBot (TeleChem Corp.). The slides were rehydrated and blocked in a solution containing $100 \mathrm{mmol} / \mathrm{l}$ ethanolamine, $1 \mathrm{~mol} / 1$ Tris ( $\mathrm{pH} 9.0$ ), and 0.1\% SDS for $20 \mathrm{~min}$ at $50^{\circ} \mathrm{C}$ and then rinsed thoroughly with water and spun dry. Purified miRNAs were labeled using a mirVana miRNA Labeling kit (Applied Biosystems, Inc.) and amine-reactive Cy5 or Cy3 dyes, according to the manufacturer's instructions. Poly(A) polymerase and a mixture of unmodified and amine-modified nucleotides were intially used to attach a polynucleotide tail to the $3^{\prime}$ end of each miRNA. The amine-modified miRNAs were then cleaned and coupled to NHS-ester modified Cy5 or Cy3 dye (Amersham Biosciences, Piscataway, NJ, USA). The RNA from normal ovarian and cancer tissues was labeled with $\mathrm{Cy} 3$ and $\mathrm{Cy} 5$ dye, respectively. Slides were hybridized for $12-16 \mathrm{~h}$ at $42^{\circ} \mathrm{C}$ in sealed cassettes under controlled humidity. Raw data were extracted using software according to the manufacturer's instructions (Agilent G4450AA Feature Extraction software 9.5). The array data were filtered with a detection P-value of $<0.05$ (similar to signal to noise) in all the samples. RNA hybridization and scanning were performed by Bohao Biotechnology Inc. (Shanghai, China). 
Table II. Clinicopathological characteristics of ovarian carcinoma cases for microarray $(n=8)$.

\begin{tabular}{lccccr}
\hline Sample no. & Age & Clinical stage & Diff grade & LNM & CA125 (U/ml) \\
\hline C1 & 58 & I & WD/MD & No & 41.77 \\
C2 & 51 & III & WD & Yes & 831.00 \\
C4 & 49 & III & MD & Yes & 670.70 \\
C5 & 53 & III & MD/PD & Yes & 1161.00 \\
C6 & 52 & IV & MD/PD & No & 670.70 \\
C7 & 75 & III & WD & Yes & 326.60 \\
C8 & 63 & IV & MD/PD & Yes & 2347.00 \\
C10 & 49 & IV & WD & No & 408.90
\end{tabular}

WD, well differentiated; MD, moderately differentiated; PD, poorly differentiated; Diff grade, differentiation grade; LNM, lymph node metastasis .

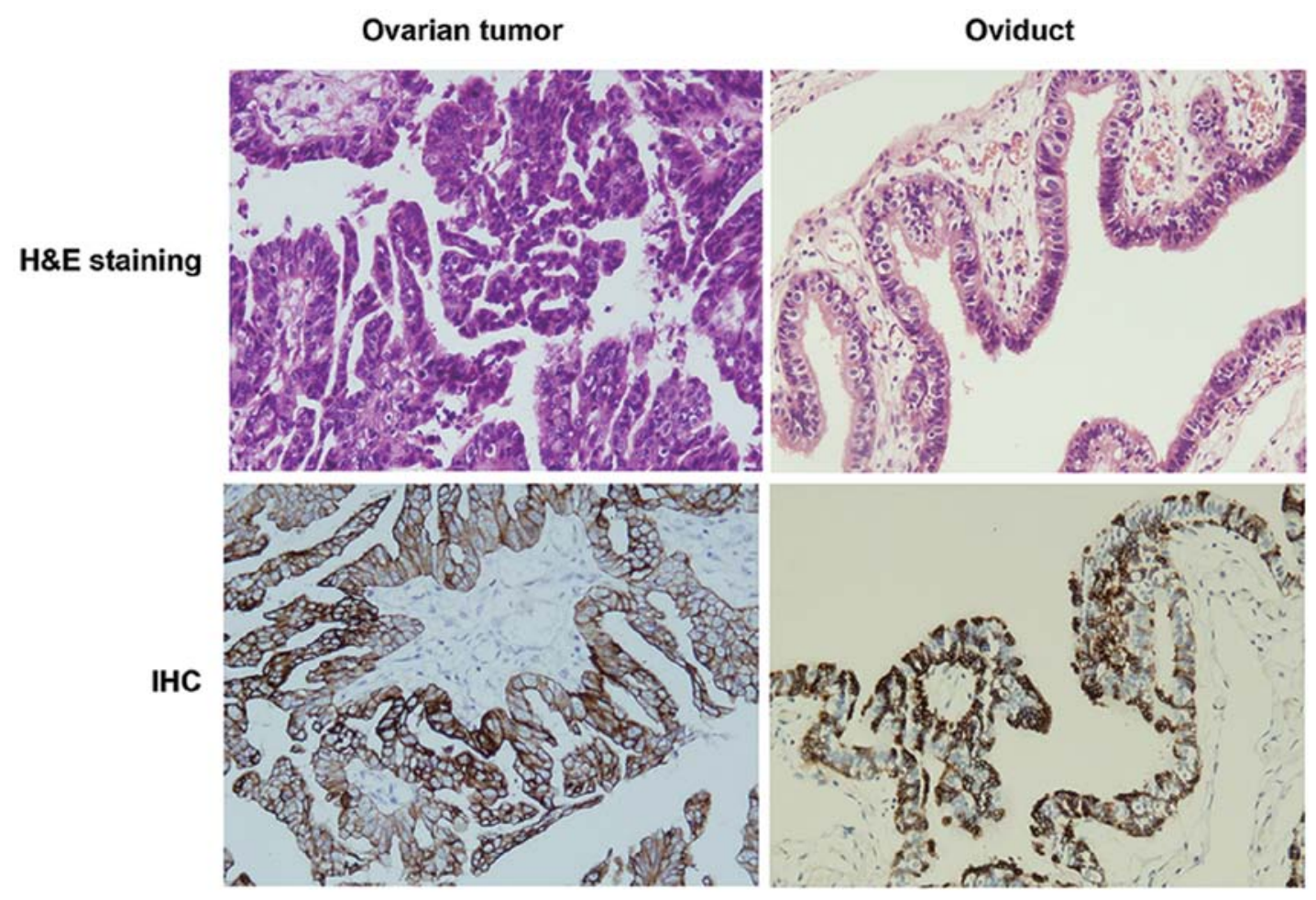

Figure 1. Hematoxylin and eosin (H\&E) staining (upper panels) and immunohistochemical analysis (lower panels) of cytokeratin 7 (CK7) expression in ovarian tumor or oviduct tissues (magnification, x200).

Quantitative polymerase chain reaction $(R T-q P C R)$. To validate the miRNA microarray findings, RT-qPCR was conducted for 100 cases of ovarian tumor and 50 cases of normal oviduct tissues. miRNA expression was analyzed using the SYBR-based stem-loop quantitative PCR method. Briefly, 100 ng of total RNA was reverse-transcribed to cDNAs with the stem-loop RT primers in a Veriti Thermal Cycler detector (Applied Biosystems, Inc.). qPCR was performed using THUNDERBIRD SYBR qPCR Mix (Toyobo, Co., Ltd., Osaka, Japan) according to the manufacturer's instructions in a CFX96 Real-time PCR Detection System (Bio-Rad, Hercules, CA, USA). The miRNA levels were normalized to U6, which was used as an internal control. The relative abundance of each miRNA was calculated using the comparative $\mathrm{Ct}\left(2^{-\Delta \Delta C t}\right)$ method, and the results were assessed by the t-test.
Bioinformatics analysis. To predict the target genes of differentially expressed miRNAs, the miRecords database (24) integrating multiple miRNA target prediction tools was employed. To reduce the false-positive results, the genes predicted by at least three tools of miRecords were selected as target genes for subsequent analysis. We subsequently used the DAVID (25) to annotate the biological function of these target genes. Then the DIANA-miRPath (26) was used to predict the top canonical pathways involving these genes.

Statistical analysis. Data were expressed as the mean \pm SD of at least three independent experiments. Group differences were compared using one-way ANOVA or the two-tailed Student's t-test from SPSS version 19.0 software (SPSS, Inc., Chicago, IL, USA). The Mann-Whitney test was conducted to compare 
Table III. Downregulated miRNAs in serous ovarian tumors.

\begin{tabular}{|c|c|c|}
\hline Name & Fold change & P-value \\
\hline hsa-miR-34b-3p & 0.000379 & $9.52 \mathrm{E}-13$ \\
\hline hsa-miR-34c-5p & 0.000774 & 2.42E-07 \\
\hline hsa-miR-34c-3p & 0.002194 & $8.82 \mathrm{E}-12$ \\
\hline hsa-miR-34b-5p & 0.007113 & $1.48 \mathrm{E}-10$ \\
\hline hsa-miR-129-1-3p & 0.009963 & $1.11 \mathrm{E}-09$ \\
\hline hsa-miR-450a-5p & 0.012179 & $5.82 \mathrm{E}-10$ \\
\hline hsa-miR-4423-3p & 0.012753 & $1.43 \mathrm{E}-07$ \\
\hline hsa-miR-542-3p & 0.018088 & $1.31 \mathrm{E}-08$ \\
\hline hsa-miR-449b-5p & 0.023804 & $1.76 \mathrm{E}-06$ \\
\hline hsa-miR-512-3p & 0.032967 & 4.03E-08 \\
\hline hsa-miR-542-5p & 0.040007 & $2.10 \mathrm{E}-05$ \\
\hline hsa-miR-139-5p_v18.0 & 0.043343 & $3.75 \mathrm{E}-05$ \\
\hline hsa-miR-503-5p & 0.059054 & 0.000124 \\
\hline hsa-miR-375 & 0.060364 & 0.000318 \\
\hline hsa-miR-1180 & 0.062304 & $3.62 \mathrm{E}-05$ \\
\hline hsa-miR-449a & 0.063076 & 0.002884 \\
\hline hsa-miR-424-5p & 0.068363 & $3.72 \mathrm{E}-09$ \\
\hline hsa-miR-23b-5p & 0.092525 & $5.83 \mathrm{E}-06$ \\
\hline hsa-miR-129-2-3p & 0.095294 & $3.30 \mathrm{E}-05$ \\
\hline hsa-miR-126-5p & 0.111644 & 0.000517 \\
\hline hsa-miR-125b-2-3p & 0.122013 & 0.001426 \\
\hline hsa-miR-3607-3p & 0.127367 & 0.000367 \\
\hline hsa-miR-135a-5p & 0.146382 & 0.004022 \\
\hline hsa-miR-374c-5p & 0.154043 & 0.000272 \\
\hline hsa-miR-328 & 0.188851 & 0.00099 \\
\hline hsa-miR-4324 & 0.208349 & 0.002182 \\
\hline hsa-miR-95 & 0.215592 & 0.003105 \\
\hline hsa-miR-99a-5p & 0.218042 & $9.51 \mathrm{E}-05$ \\
\hline hsa-miR-92b-3p & 0.21966 & 0.001364 \\
\hline hsa-miR-139-3p & 0.221399 & 0.00206 \\
\hline hsa-miR-505-5p & 0.243588 & 0.000854 \\
\hline hsa-miR-145-3p & 0.245729 & 0.004879 \\
\hline hsa-miR-548aa & 0.257712 & 0.002667 \\
\hline hsa-miR-195-5p & 0.265545 & 0.000422 \\
\hline hsa-miR-497-5p & 0.273068 & 0.000383 \\
\hline hsa-miR-769-5p & 0.279618 & 0.007579 \\
\hline hsa-miR-338-5p & 0.280438 & 0.001696 \\
\hline hsa-miR-424-3p & 0.287992 & 0.009064 \\
\hline hsa-miR-361-3p & 0.291801 & $6.64 \mathrm{E}-06$ \\
\hline hsa-miR-100-5p & 0.293041 & 0.000155 \\
\hline hsa-miR-885-5p & 0.300037 & 0.000821 \\
\hline hsa-miR-548d-5p & 0.301616 & 0.007974 \\
\hline hsa-miR-744-5p & 0.305494 & 0.005764 \\
\hline hsa-miR-4657 & 0.31529 & 0.003572 \\
\hline hsa-miR-140-3p & 0.319548 & $1.31 \mathrm{E}-05$ \\
\hline hsa-miR-625-5p & 0.332339 & 0.00451 \\
\hline hsa-miR-339-3p & 0.337476 & 0.000738 \\
\hline hsa-miR-423-3p & 0.340606 & 0.002225 \\
\hline hsa-miR-4731-3p & 0.349857 & 0.005428 \\
\hline hsa-miR-31-5p & 0.362345 & 0.003684 \\
\hline hsa-miR-30a-5p & 0.366509 & 0.000236 \\
\hline hsa-miR-598 & 0.380527 & 0.006472 \\
\hline
\end{tabular}

Table III. Continued.

\begin{tabular}{lll}
\hline hsa-let-7c & 0.385549 & $2.86 \mathrm{E}-05$ \\
hsa-miR-145-5p & 0.386009 & 0.008751 \\
hsa-miR-140-5p & 0.388593 & 0.000112 \\
hsa-miR-29c-5p & 0.389239 & 0.000209 \\
hsa-miR-125b-5p & 0.392979 & 0.003191 \\
hsa-miR-423-5p & 0.40677 & $1.16 \mathrm{E}-06$ \\
hsa-miR-1229-3p & 0.428179 & 0.004909 \\
hsa-miR-126-3p & 0.432629 & 0.000745 \\
hsa-miR-3653 & 0.476556 & $1.87 \mathrm{E}-05$ \\
hsa-miR-664a-3p & 0.494524 & $1.88 \mathrm{E}-05$ \\
hsa-miR-101-3p & 0.497294 & 0.00134 \\
\hline
\end{tabular}

miRNA expression according to the clinicopathological parameters. $\mathrm{P}<0.05$ was considered to be statistically significant.

\section{Results}

Clinicopathological characteristics of patients. In total, 100 serous ovarian cancer patients were enrolled in the study (Table I). Only patients who did not receive neoadjuvant chemotherapy were recruited. Their age ranged from 21 to 90 years with 25 patients $(25 \%)$ aged $>60$ years. The clinical stages of ovarian carcinoma following initial diagnosis were as follows: low stages (I,II) in 28 cases $(28 \%)$ and high stages (III,IV) in 72 cases $(72 \%)$. Differentiated grading for ovarian carcinoma was classified into three grades, of which eight cases were well-differentiated and 92 cases were moderately/poorly differentiated. Lymph node metastasis was detected in 42 cases. Carbohydrate antigen-125 (CA125) is the most frequently used biomarker for ovarian cancer detection (27). Of 100 carcinomas analyzed, the serum CA125 value in 70 cases was $>200 \mathrm{U} / \mathrm{ml}$.

$H \& E$ staining and IHC analysis ovarian tumor and normal oviduct tissues. To confirm the histologic features of ovarian tumor and normal oviduct tissues, H\&E staining was performed. As shown in Fig. 1, the H\&E staining showed that signet ring-like cells were diffused in ovarian tumor tissues. We then performed IHC to examine the characteristics of ovarian tumor by $\mathrm{CK} 7$, which is considered to be a useful discriminant marker to differentiate primary epithelial ovarian tumors from tumors metastatic to the ovary (28). In contrast to the normal oviduct tissues, the ovarian tumors were stained strongly for CK7 expression (Fig. 1).

MiRNA-expressed profiling by microarray analysis. To identify miRNAs differentially expressed in serous ovarian cancer compared with the corresponding normal oviduct tissues, each eight cases of serous ovarian tumors and oviduct tissue specimens were randomly selected and assessed using a customized miRNA microarray. The clinicopathological characteristics of the eight ovarian tumor patients are shown in Table II. After performing fold change filtering (fold-change $\geq 2$ ) on the differentially expressed miRNAs, we found that 63 miRNAs were downregulated (Table III) and 43 miRNAs were upregu- 
Table IV. Upregulated miRNAs in serous ovarian tumors.

\begin{tabular}{|c|c|c|}
\hline Name & Fold change & P-value \\
\hline hsa-miR-452-5p & 38.48964 & 0.000413 \\
\hline hsa-miR-409-3p & 15.07982 & 0.000534 \\
\hline hsa-miR-224-5p & 14.41281 & 0.001068 \\
\hline hsa-miR-382-5p & 13.06539 & 0.003336 \\
\hline hsa-miR-4688 & 10.7648 & 0.00013 \\
\hline hsa-miR-4738-3p & 8.289778 & 0.000545 \\
\hline hsa-miR-4535 & 7.585281 & 0.000439 \\
\hline hsa-miR-877-5p & 6.691364 & 0.000272 \\
\hline hsa-miR-601 & 6.207081 & 0.000241 \\
\hline hsa-miR-202-3p & 6.1395 & 0.002417 \\
\hline hsa-miR-370 & 6.013743 & 0.001873 \\
\hline hsa-miR-135b-5p & 5.567153 & 0.002722 \\
\hline hsa-miR-3676-5p & 5.427661 & 0.000597 \\
\hline hsa-miR-99b-3p & 5.386582 & 0.004613 \\
\hline hsa-miR-1226-5p & 4.510622 & 0.002622 \\
\hline hsa-miR-4476 & 4.475274 & 0.001844 \\
\hline hsa-miR-1185-2-3p & 4.44722 & 0.007731 \\
\hline hsa-miR-663a & 4.042133 & 0.003116 \\
\hline hsa-miR-4417 & 3.913188 & $6.08 \mathrm{E}-05$ \\
\hline hsa-miR-4776-5p & 3.836805 & 0.004647 \\
\hline hsa-miR-4741 & 3.52447 & 0.001585 \\
\hline hsa-miR-1202 & 3.464635 & 0.000311 \\
\hline hsa-miR-3960 & 3.419049 & 7.91E-05 \\
\hline hsa-miR-4634 & 2.890565 & 0.000611 \\
\hline hsa-miR-4687-3p & 2.579342 & 0.000305 \\
\hline hsa-miR-3196 & 2.50828 & 0.001451 \\
\hline hsa-miR-4281 & 2.439574 & 0.000739 \\
\hline hsa-miR-1207-5p & 2.391054 & $8.46 \mathrm{E}-05$ \\
\hline hsa-miR-4539 & 2.367196 & 0.00059 \\
\hline hsa-miR-21-5p & 2.365285 & 0.000222 \\
\hline hsa-miR-1225-5p & 2.309348 & 0.000653 \\
\hline hsa-miR-939-5p & 2.26632 & 0.008186 \\
\hline hsa-miR-1185-1-3p & 2.254424 & 0.000415 \\
\hline hsa-miR-27a-3p & 2.252088 & 0.003182 \\
\hline hsa-miR-483-5p & 2.225036 & 0.000655 \\
\hline hsa-miR-575 & 2.147543 & 0.003077 \\
\hline hsa-miR-4739 & 2.146543 & 0.000172 \\
\hline hsa-miR-940 & 2.135077 & 0.007481 \\
\hline hsa-miR-642b-3p & 2.10437 & 0.005702 \\
\hline hsa-miR-4530 & 2.071332 & 0.006489 \\
\hline hsa-miR-3663-3p & 2.069014 & 0.00113 \\
\hline hsa-miR-134 & 2.063351 & $1.58 \mathrm{E}-05$ \\
\hline hsa-miR-1290 & 2.004518 & 0.003804 \\
\hline
\end{tabular}

lated (Table IV) in serous ovarian cancers compared with control tissues $(\mathrm{P} \leq 0.05)$. The heat map (Fig. 2) shows the results of the unsupervised hierarchical clustering based on the significantly differentially expressed miRNAs listed in Tables II and III.

Validation of unique miRNAs by RT-qPCR. To confirm the miRNA expression pattern obtained from microarray analysis, RT-qPCR was used to quantify the expression levels of specific miRNAs from the 100 cases of serous ovarian tumors and 50 cases of normal oviduct tissue specimens. In total,

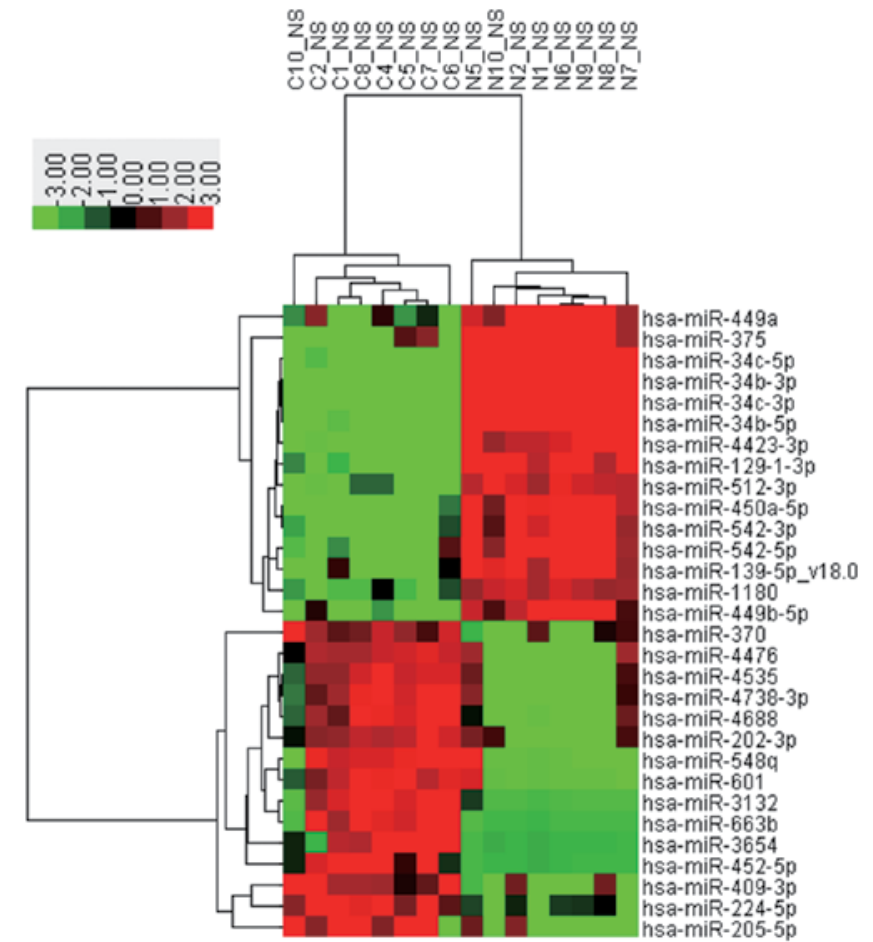

Figure 2. Heat map and hierarchical clustering analysis of microRNAs (miRNAs) that exhibited a $\geq 2$-fold increase or decrease in ovarian cancer compared with normal oviduct tissues. C1, C2, C4, C5, C6, $\mathrm{C} 7, \mathrm{C} 8$ and $\mathrm{C} 10$ are the ovarian cancer samples, whereas $\mathrm{N} 1, \mathrm{~N} 2, \mathrm{~N} 5, \mathrm{~N} 6$, $\mathrm{N} 7, \mathrm{~N} 8, \mathrm{~N} 9$ and $\mathrm{N} 10$ are the oviduct tissue samples. The expression level of miRNA is color-coded. Red, higher miRNA expression; green, lower miRNA expression; black, no difference.

five (miR-129-1-3p, miR-542-5p, miR-450a-5p, miR-129-2-3p and miR-424-5p) of the 106 miRNAs that were differentially expressed in ovarian tumors were selected for subsequent validation by RT-qPCR. These miRNAs were selected due to their significantly fold changes $(>10)$ in expression levels in ovarian tumors as compared with normal oviduct tissues. Additionally, we would like to identify research candidates for future research. The RT-qPCR results demonstrated that all five miRNAs were markedly downregulated in serous ovarian tumors as compared with control oviduct tissues (Fig. 3, $\mathrm{P}<0.01$, which was consistent with the results of the microarray analysis.

Gene ontology terms and canonical pathway annotation of miRNA targets. As miRNAs play critical roles in post-transcriptional regulation by targeting mRNAs, we retrieved the putative target genes of differentially expressed miRNAs using the miRecords database (24) and selected the target genes retrieved by at least three tools contained in miRecords. To examine the function of the differentially expressed miRNAs, we collected the top $25 \%$ of the predicted miRNA targets and performed gene ontology (GO) term and pathway annotation. GO term annotation results showed that the regulation of transcription, RNA splicing, mRNA processing and cell cycle were the most significantly enriched GO terms (Fig. 4A). This finding suggested that miRNAs may regulate transcription factors or cell cycle-related genes. The canonical pathways predicted to be controlled by the dysregulated miRNAs were also assessed. The top 10 pathways are shown in Fig. 4B. 
Table V. Correlation between clinicopathological parameters and miRNA fold change of ovarian serous carcinoma $(\mathrm{n}=100)$.

\begin{tabular}{|c|c|c|c|c|c|c|c|c|c|c|c|}
\hline \multirow[b]{2}{*}{ Parameters } & \multirow[b]{2}{*}{ No. } & \multicolumn{2}{|c|}{ miR-129-1-3p } & \multicolumn{2}{|c|}{ miR-542-5p } & \multicolumn{2}{|c|}{ miR-450a-5p } & \multicolumn{2}{|c|}{ miR-129-2-3p } & \multicolumn{2}{|c|}{ miR-424-5p } \\
\hline & & $\begin{array}{l}\text { Fold } \\
\text { change }\end{array}$ & P-value & $\begin{array}{l}\text { Fold } \\
\text { change }\end{array}$ & P-value & $\begin{array}{l}\text { Fold } \\
\text { change }\end{array}$ & P-value & $\begin{array}{l}\text { Fold } \\
\text { change }\end{array}$ & P-value & $\begin{array}{l}\text { Fold } \\
\text { change }\end{array}$ & P-value \\
\hline \multicolumn{12}{|l|}{ FIGO grade } \\
\hline 1 & 8 & 0.02 & 0.23 & 0.11 & 0.35 & 0.18 & 0.12 & 0.03 & 0.89 & 0.38 & 0.56 \\
\hline 2 & 76 & 0.03 & & 0.20 & & 0.20 & & 0.05 & & 0.22 & \\
\hline 3 & 16 & 0.04 & & 0.16 & & 0.10 & & 0.05 & & 0.21 & \\
\hline \multicolumn{12}{|l|}{ Clinical stage } \\
\hline Low (I/II) & 26 & 0.02 & 0.66 & 0.14 & 0.16 & 0.17 & 0.70 & 0.03 & 0.37 & 0.22 & 0.92 \\
\hline High (III/IV) & 74 & 0.04 & & 0.20 & & 0.19 & & 0.05 & & 0.23 & \\
\hline \multicolumn{12}{|l|}{ Lymph node } \\
\hline Negative & 57 & 0.03 & 0.14 & 0.16 & 0.20 & 0.19 & 0.10 & 0.04 & 0.62 & 0.23 & 0.90 \\
\hline Positive & 43 & 0.04 & & 0.21 & & 0.17 & & 0.05 & & 0.23 & \\
\hline \multicolumn{12}{|c|}{ Distant metastasis } \\
\hline Negative & 82 & 0.04 & 0.43 & 0.17 & 0.26 & 0.17 & 0.57 & 0.05 & 0.74 & 0.21 & 0.30 \\
\hline Positive & 18 & 0.03 & & 0.24 & & 0.21 & & 0.04 & & 0.31 & \\
\hline
\end{tabular}

FIGO, International Federation of Gynecology and Obstetrics.

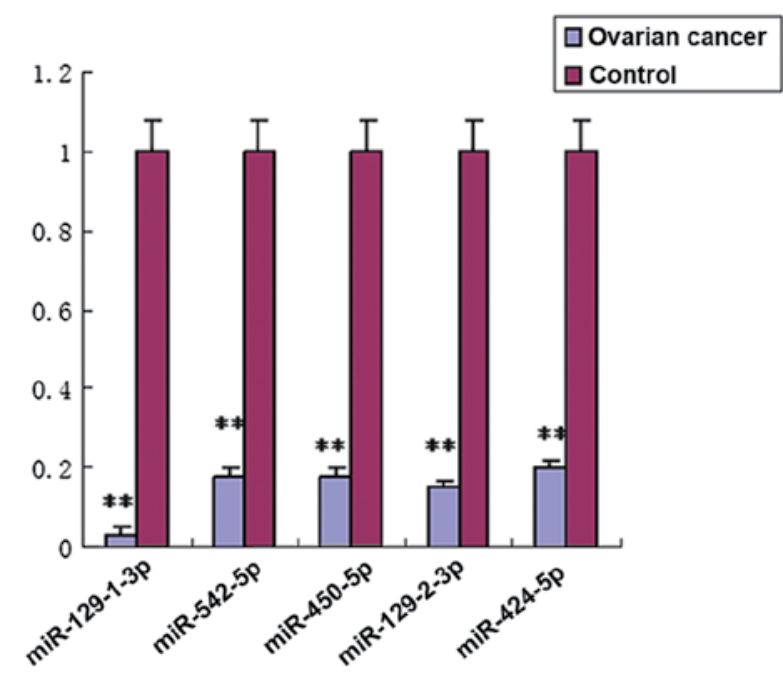

Figure 3. Quantitative polymerase chain reaction (qRT-PCR) analysis of five microRNA (miRNA) expression in samples from ovarian cancer groups or control oviduct tissue groups. Data are presented as means \pm SD from three individual experiments. ${ }^{* *}$ Statistical significance at $\mathrm{P}<0.01$.

The majority of pathways have been shown to be involved in carcinogenesis, including the mTOR, Wnt, TGF- $\beta$ and MAPK signaling pathways, which may demonstrate the possible roles and mechanisms of these differentially expressed miRNAs in ovarian development or metastasis.

Correlation of miRNA expression with clinicopathological parameters. The relationship between miRNA expression and clinicopathological parameters, including FIGO grade, clinical stage, lymph node and distant metastasis, were evaluated in serous ovarian carcinomas (Table V). The downregulation

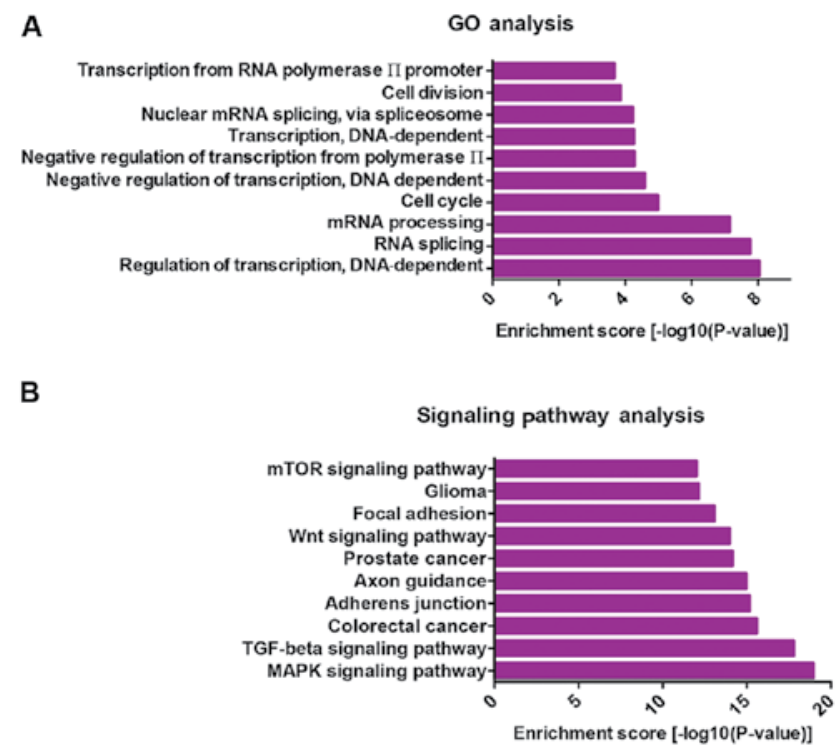

Figure 4. (A) Top 10 functional gene ontology terms of the predicted microRNA (miRNA) target genes. (B) Top 10 signaling pathways predicted to be regulated by the differentially expressed miRNAs.

of miR-450a-5p tended to be associated with FIGO grade or lymph node metastasis; however, this was not statistically significant $(\mathrm{P}=0.12$ or 0.10$)$. No significant relationship was observed between miRNA expression and clinical stage or distant metastasis.

\section{Discussion}

Serous ovarian carcinoma is the most common histological subtype of ovarian cancer. The majority of patients receiving pathological diagnosis at stage I survive, whereas patients 
receiving diagnosis at an advanced stage succumb to the disease. Currently, serous ovarian carcinoma is characterized by late diagnosis, rapid progression and poor prognosis (4). A number of studies have suggested that miRNAs play a vital role in the identification of gene expression patterns for use as a diagnostic and prognostic tool for cancer patients and may serve as molecular targets for therapy.

In the present study, we have demonstrated that 63 miRNAs were significantly downregulated and 43 miRNAs were upregulated in serous ovarian carcinoma compared with normal oviduct tissues. This miRNA pattern includes some well-known tumor suppressor miRNAs (miR-34b, miR-34c, miR-375 and let-7c) and oncomiRs (miR-370, miR-21). MiR-34b/c was reported to suppress cell proliferation and migration and was a predictive marker of a number of tumors (29-31). MiR-375 inhibits cell growth, migration and invasion and functions as a tumor suppressor in various types of cancer $(32,33)$. MiR-21 detection has a prognostic value in patients with cancer, particularly in head and neck squamous cell carcinoma and digestion system cancers (34). These miRNAs are involved in the processes of cell proliferation, migration/invasion, and play important roles in cancer progression, suggesting that these miRNAs may be useful markers for the differentiation of malignant tumors from borderline tumors.

Five (miR-129-1-3p, miR-542-5p, miR-450a-5p, miR-129-2-3p and miR-424-5p) of the 106 miRNAs identified in the present study were validated by RT-qPCR, and their expression was confirmed to be significantly consistent with the microarray data. The results indicated that the differential miRNA pattern in serous ovarian carcinoma as compared with normal oviduct tissues was relatively credible. Furthermore, these five miRNAs identified in the current study have not been detected previously in ovarian carcinomas. MiR-129 has been shown to act as a tumor suppressor in gastric cancer (35), colorectal cancer (36) or hepatocellular carcinoma (37). MiR-542-5p was first reported as a novel tumor suppressor in neuroblastoma (38). Downregulation of miR-450a was demonstrated to be associated with poor prognosis in esophageal squamous cell carcinoma (39). However, miR-424-5p was reported to be frequently upregulated in pancreatic cancer and suppress the expression of SOCS6 (40), which was not in agreement with our finding that miR-424-5p was downregulated in ovarian cancer. The reason for this discrepancy may be that miRNA was differentially expressed in different cancer types. Our results suggest that these miRNAs are likely promising diagnostic and prognostic biomarkers in serous ovarian carcinomas.

Of note, single miRNAs can exert different functions by targeting multiple mRNAs, and a single mRNA can be regulated by multiple miRNAs (6). To investigate the function of the differentially expressed miRNAs, we performed GO terms and canonical pathway annotation of miRNA targets. Functional analysis revealed that the GO category of the cell cycle was significantly dysregulated in ovarian cancer. The cell cycle, which is modulated by a number of regulators, including cyclins and cyclin-dependent kinases, is crucial for the life cycle of mammals. Cell cycle dysregulation is involved in many diseases, including cancer (41). Our results reveal that the cell cycle plays an important role in the progression of ovarian cancer, which is consistent with previous studies $(42,43)$. The pathway analysis demonstrated that the miRNAs extracted in the present study control several pathways relevant for the regulation of ovarian cancer. The MAPK and PI3K-Akt-mTOR signaling pathways have been shown to be involved in estrogen-dependent gynecological disorders including polycystic ovarian syndrome (42). It was reported that TGF- $\beta$ acts to inhibit proliferation of normal ovarian surface epithelium and early stage ovarian carcinoma (43). Our results indicate that the differentially expressed miRNAs may function in ovarian cancer through the modulation of these signaling pathways.

It has also been demonstrated that miRNA expression profiles are correlated to clinicopathological parameters of human cancers, including clinical stage, FIGO grade and lymph node involvement (44). In this study, we have shown that downregulation of miR-450a-5p tended to be associated with FIGO grade or lymph node metastasis, although this association was not statistically significant $(\mathrm{P}=0.12$ or 0.10$)$. Additionally, the expression of miRNAs was not significantly correlated with clinical stage or distant metastasis. The reason may be that these miRNAs were not directly associated with the aforementioned chinicopathological factors. Thus, further investigations should be conducted to confirm this lack of association.

In conclusion, we identified 106 miRNAs that were aberrantly expressed in serous ovarian carcinoma as compared to normal oviduct tissues, suggesting that these miRNAs are involved in ovarian tumorigenesis and therefore may be used as prognostic markers. Future studies are required to validate the miRNA targets and elucidate the mechanism of miRNA function during ovarian tumorigenesis.

\section{Acknowledgements}

This study was supported by grants funded by the Chinese Government, HG3310 and 2007K09-09.

\section{References}

1. Permuth-Wey J and Sellers TA: Epidemiology of ovarian cancer. Methods Mol Biol 472: 413-437, 2009.

2. Seidman JD and Kurman RJ: Pathology of ovarian carcinoma. Hematol Oncol Clin North Am 17: 909-925, vii, 2003

3. Shih IeM and Kurman RJ: Ovarian tumorigenesis: a proposed model based on morphological and molecular genetic analysis. Am J Pathol 164: 1511-1518, 2004.

4. Seidman JD, Horkayne-Szakaly I, Haiba M, Boice CR, Kurman RJ and Ronnett BM: The histologic type and stage distribution of ovarian carcinomas of surface epithelial origin. Int J Gynecol Pathol 23: 41-44, 2004.

5. Levanon K, Crum C and Drapkin R: New insights into the pathogenesis of serous ovarian cancer and its clinical impact. J Clin Oncol 26: 5284-5293, 2008.

6. Bartel DP: MicroRNAs: genomics, biogenesis, mechanism, and function. Cell 116: 281-297, 2004.

7. Wang F, Niu G, Chen $X$ and Cao F: Molecular imaging of microRNAs. Eur J Nucl Med Mol Imaging 38: 1572-1579, 2011.

8. Calin GA and Croce CM: MicroRNA signatures in human cancers. Nat Rev Cancer 6: 857-866, 2006.

9. Wang F, Fu XD, Zhou Y and Zhang Y: Down-regulation of the cyclin E1 oncogene expression by microRNA-16-1 induces cell cycle arrest in human cancer cells. BMB Rep 42: 725-730, 2009.

10. Wang F, Song X, Li X, et al: Noninvasive visualization of microRNA-16 in the chemoresistance of gastric cancer using a dual reporter gene imaging system. PLoS One 8: e61792, 2013.

11. Resnick KE, Alder H, Hagan JP, Richardson DL, Croce CM and Cohn DE: The detection of differentially expressed microRNAs from the serum of ovarian cancer patients using a novel real-time PCR platform. Gynecol Oncol 112: 55-59, 2009. 
12. Yu SL, Chen HY, Chang GC, et al: MicroRNA signature predicts survival and relapse in lung cancer. Cancer Cell 13: 48-57, 2008.

13. Zaman MS, Maher DM, Khan S, Jaggi M and Chauhan SC: Current status and implications of microRNAs in ovarian cancer diagnosis and therapy. J Ovarian Res 5: 44, 2012.

14. Zhang L, Volinia S, Bonome T, et al: Genomic and epigenetic alterations deregulate microRNA expression in human epithelial ovarian cancer. Proc Natl Acad Sci USA 105: 7004-7009, 2008.

15. Iorio MV, Visone R, Di Leva G, et al: MicroRNA signatures in human ovarian cancer. Cancer Res 67: 8699-8707, 2007.

16. Nam EJ, Yoon H, Kim SW, et al: MicroRNA expression profiles in serous ovarian carcinoma. Clin Cancer Res 14: 2690-2695, 2008.

17. Gallagher MF, Flavin RJ, Elbaruni SA, et al: Regulation of microRNA biosynthesis and expression in 2102Ep embryonal carcinoma stem cells is mirrored in ovarian serous adenocarcinoma patients. J Ovarian Res 2: 19, 2009.

18. Wyman SK, Parkin RK, Mitchell PS, et al: Repertoire of microRNAs in epithelial ovarian cancer as determined by next generation sequencing of small RNA cDNA libraries. PLoS One 4: e5311, 2009.

19. Laios A, O'Toole S, Flavin R, et al: Potential role of miR-9 and miR-223 in recurrent ovarian cancer. Mol Cancer 7: 35, 2008.

20. Eitan R, Kushnir M, Lithwick-Yanai G, et al: Tumor microRNA expression patterns associated with resistance to platinum based chemotherapy and survival in ovarian cancer patients. Gynecol Oncol 114: 253-259, 2009.

21. Yang N, Kaur S, Volinia S, et al: MicroRNA microarray identifies Let-7i as a novel biomarker and therapeutic target in human epithelial ovarian cancer. Cancer Res 68: 10307-10314, 2008.

22. Mezzanzanica D, Bagnoli M, De Cecco L, Valeri B and Canevari S: Role of microRNAs in ovarian cancer pathogenesis and potential clinical implications. Int J Biochem Cell Biol 42: 1262-1272, 2010.

23. Kunej T, Godnic I, Ferdin J, Horvat S, Dove P and Calin GA: Epigenetic regulation of microRNAs in cancer: an integrated review of literature. Mutat Res 717: 77-84, 2011.

24. Xiao F, Zuo Z, Cai G, Kang S, Gao X and Li T: miRecords: an integrated resource for microRNA-target interactions. Nucleic Acids Res 37: D105-D110, 2009.

25. Huang da W, Sherman BT and Lempicki RA: Systematic and integrative analysis of large gene lists using DAVID bioinformatics resources. Nat Protoc 4: 44-57, 2009.

26. Vlachos IS,Kostoulas N, Vergoulis T, et al: DIANA miRPath v.2.0 investigating the combinatorial effect of microRNAs in pathways. Nucleic Acids Res 40: W498-W504, 2012.

27. Suh KS, Park SW, Castro A, et al: Ovarian cancer biomarkers for molecular biosensors and translational medicine. Expert Rev Mol Diagn 10: 1069-1083, 2010.

28. Kriplani D and Patel MM: Immunohistochemistry: a diagnostic aid in differentiating primary epithelial ovarian tumors and tumors metastatic to the ovary. South Asian J Cancer 2: 254-258, 2013

29. Muraoka T, Soh J, Toyooka S, et al: The degree of microRNA-34b/c methylation in serum-circulating DNA is associated with malignant pleural mesothelioma. Lung Cancer 82: 485-490, 2013.
30. Nadal E, Chen G, Gallegos M, et al: Epigenetic inactivation of microRNA-34b/c predicts poor disease-free survival in early-stage lung adenocarcinoma. Clin Cancer Res 19: 6842-6852, 2013

31. Suzuki R, Yamamoto E, Nojima M, et al: Aberrant methylation of microRNA-34b/c is a predictive marker of metachronous gastric cancer risk. J Gastroenterol: Aug 13, 2013 (Epub ahead of print).

32. He XX, Chang Y, Meng FY, et al: MicroRNA-375 targets AEG-1 in hepatocellular carcinoma and suppresses liver cancer cell growth in vitro and in vivo. Oncogene 31: 3357-3369, 2012.

33. Kong KL, Kwong DL, Chan TH, et al: MicroRNA-375 inhibit tumour growth and metastasis in oesophageal squamous cell carcinoma through repressing insulin-like growth factor 1 receptor. Gut 61: 33-42, 2012.

34. Fu X, Han Y, Wu Y, et al: Prognostic role of microRNA-21 in various carcinomas: a systematic review and meta-analysis. Eur J Clin Invest 41: 1245-1253, 2011.

35. Yu X, Song H, Xia T, et al: Growth inhibitory effects of three miR-129 family members on gastric cancer. Gene 532: 87-93, 2013

36. Karaayvaz M, Zhai $\mathrm{H}$ and Ju J: miR-129 promotes apoptosis and enhances chemosensitivity to 5-fluorouracil in colorectal cancer. Cell Death Dis 4: e659, 2013.

37. Liu Y, Hei Y, Shu Q, et al: VCP/p97, down-regulated by microRNA-129-5p, could regulate the progression of hepatocellular carcinoma. PLoS One 7: e35800, 2012.

38. Bray I, Tivnan A, Bryan K, et al: MicroRNA-542-5p as a novel tumor suppressor in neuroblastoma. Cancer Lett 303: 56-64, 2011.

39. Yamamoto S, Inoue J, Kawano T, Kozaki K, Omura K and Inazawa J: The impact of mirna-based molecular diagnostics and treatment of NRF2-stabilized tumors. Mol Cancer Res 12: 58-68, 2014.

40. Wu K, Hu G, He X, et al: MicroRNA-424-5p suppresses the expression of SOCS6 in pancreatic cancer. Pathol Oncol Res 19: 739-748, 2013.

41. Liang LH and He XH: Macro-management of microRNAs in cell cycle progression of tumor cells and its implications in anti-cancer therapy. Acta Pharmacol Sin 32: 1311-1320, 2011

42. Makker A, Goel MM, Das V and Agarwal A: PI3K-Akt-mTOR and MAPK signaling pathways in polycystic ovarian syndrome, uterine leiomyomas and endometriosis: an update. Gynecol Endocrinol 28: 175-181, 2012.

43. Nilsson EE and Skinner MK: Role of transforming growth factor beta in ovarian surface epithelium biology and ovarian cancer. Reprod Biomed Online 5: 254-258, 2002.

44. Slaby O, Svoboda M, Fabian P, et al: Altered expression of miR-21, miR-31, miR-143 and miR-145 is related to clinicopathologic features of colorectal cancer. Oncology 72: 397-402, 2007. 